\title{
Optimal Feeder Flow Control for Grid Connected Microgrids
}

\author{
Eduard Bullich-Massaguéa,*, Mònica Aragüés-Peñalba ${ }^{a}$, Eduardo \\ Prieto-Araujo $^{\mathrm{a}}$, Andreas Sumper $^{\mathrm{a}}$, Raphael Caire ${ }^{\mathrm{b}}$ \\ ${ }^{a}$ Centre d'Innovació Tecnològica en Convertidors Estàtics i Accionamients \\ (CITCEA-UPC), Department d'Enginyeria Elèctrica, Universitat Politècnica de \\ Catalunya, UPC. Av. Diagonal 647, Pl. 2. 08028, Barcelona, Spain \\ ${ }^{b}$ Univ. Grenoble Alpes, CNRS, Grenoble INP, G2Elab, 38000 Grenoble, France.
}

\begin{abstract}
The optimal management of distributed energy resources is one of the existing challenges for the deployment of microgrids. When microgrids operate under feeder flow control mode, trying to maintain a constant selfconsumption, generators adapt their output power to compensate load and non-dispatchable generation variations. So, due to the uncertainty, finding the optimal operation point is an important task that can become complex. This paper proposes an optimal power flow problem formulation for feeder flow controlled microgrids. It is formulated as a mixed integer second order cone program considering the optimal power flow equations in its relaxed form and uncertainty by means of stochastic formulation. In addition, an algorithm is developed in order to find a feasible optimum solution of the original non-relaxed problem. The proposed algorithm can also be used in other optimal power flow problems with the condition that they must use the same relaxation. The algorithm is validated through the IEEE 33-Bus distribution test system.
\end{abstract}

Keywords:

Microgrids, optimal power flow, distributed energy resources, feeder flow, SOCP

\footnotetext{
*Corresponding author

Email address: eduard.bullich@citcea.upc.edu (Eduard Bullich-Massagué)
} 


\section{Introduction}

Wind and photovoltaic (PV) power installations are experiencing a rapid growth worldwide [1, 2]. Due to the distributed location of the resource, the conventional electric system based on large and centralized generators is, Energy Sources (DRES) and Distributed Energy Resources (DER). At the distribution level, grids have been traditionally operated as passive systems. But the integration of DRES is pushing the transformation of these networks into active systems with bidirectional power flows. New concepts are required structures are based on microgrids 3 ] or even on local energy communities.

The challenge of integrating large amount of PV and wind power generation into the electric power system is related to its non-dispatchable nature. It has been identified that it could compromise the stability of the system ation of the power grids. For instance, for large scale PV plants connected to transmission system, grid codes are being updated requiring to provide voltage, frequency and fault support [6, 7]. At distribution level, microgrids could also provide ancillary services to the external grids if DERs are in20 stalled 3 . In this direction, the control of the active power exchange with the external grid, also known as Feeder Flow Control (FFC), can help grid operators to plan their distribution grid operation. As stated in [8], from the utility's point of view, it is desirable if a portion of their networks consume constant electric power as scheduled or commanded. In essence, a feeder flow controlled microgrid is a true dispatchable system [9].

The FFC in microgrids has recently been studied in [8, 10 15]. In [8, the minimum feeder flow setpoint at the connection point of the microgrid is determined and an algorithm is developed to comply with the setpoint, but its optimal operation is not considered. In [10], the FFC under differthe transition mode) is studied. In addition, a method to determine the frequency - feeder flow droop constants is defined in order to avoid higher frequency deviations during transition (grid connected-islanded) mode. One important conclusion is that multiple Distributed Generators (DG) operating But again, the optimal economic operation is not considered. In [11] and [12], a centralized feeder flow controller based on a fuzzy PI controller to minimize 
the power deviations during various load changes is proposed. The proposed scheme consists on all DGs operating under Unit output Power Control mode (UPC), and the centralized feeder flow controller modifies each active power setpoint to maintain the desired feeder flow at the microgrid point of connection. In [13], an economic dispatch optimization problem is formulated considering a microgrid based on different control areas, where each control area performs a FFC. The objective is to minimize the cost ensuring the best of our knowledge, no other studies propose an economic dispatch optimization for a microgrid operating under FFC mode.

The economic optimization of the power systems operation can be performed considering the power flow equations (optimal power flow) or without them (optimal economic dispatch). The optimal economic dispatch for feeder flow controlled microgrids has been studied in [13], where the presence of FFC generators has been modelled as single power flow constraints in the controlled lines. This formulation does not model the behaviour when the FFC generator reaches its maximum or minimum power output. When FFC generators reach their power limits, the branch flow is no longer controlled. Note that modelling correctly the FFC generators is required when stochastic optimization is used. This is because the feeder flow setpoint is the same for different scenarios, and in some scenarios the generation may reach its limits. On the other hand, to the best of our knowledge, the optimal power flow for these grids has not been formulated before. To find a global optimum for the optimal power flow problem in radial grids, relaxation techniques have been used, leading to second order cone problems (SOCP) [16]. In addition, conditions for the objective function have been found to ensure the relaxed problem holds [16]. When these conditions are not accomplished, the optimal solution has no physical sense. To solve it, an iterative algorithm is suggested in [17], where some terms of the objective function are moved to an additional constraint to make the relaxation hold, but under some conditions the algorithm can converge to a local optimum. This happens because the optimal power flow formulation does not consider the real cost function. Traditionally, economic dispatch algorithms or optimal power flows have been formulated taking into account that generators operate under UPC mode (constant active power setpoint) [18]. So, the optimal power setpoints that minimize the cost will be maintained constant. In the FFC mode, the power generated will depend on the output of the optimization algorithm but also on the load and non-dispatchable generators variations. So, when a load vari- 
ation occurs the dispatchable generators outputs will vary and the optimal operating point can be lost.

The present paper addresses the before described problems. In particular, the contributions of this paper are:

i) The formulation an optimization problem for feeder flow controlled microgrids including the power flow equations: The problem is based on a mixed integer second order cone programming (MISOCP). The proposed formulation includes an accurate model of the feeder flow controlled generators and lines. With a set of linear constraints, the FFC generators maintain the controlled branch flows constant until they reach the maximum or minimum power output. At this moment, the proposed constraints allow the branch power flow to exceed the given setpoint.

ii) Analysis of possible improvements by using the stochastic optimisation: The present paper compares two optimization approaches namely 1-scenario optimization (non-stochastic) and n-scenario optimization (stochastic). In this case, 10 scenario optimization has been used. It is found that the stochastic approach not only can mitigate voltage limit violations due to uncertainties but also can improve the objective function by achieving a better fulfilment of the feeder flow setpoints.

iii) The development of an alternative algorithm for obtaining a feasible optimum solution from the relaxed optimal power flow problem. The proposed algorithm maintains all the cost terms in the objective function, being able to find better solutions than in other proposals. The solution method proposed in this paper is compared with the solution method proposed in [17].

While this paper presents the formulation and resolution of an optimal power flow algorithm, it does not study the mechanisms of how the setpoints of power exchange between the microgrid and the external grid are determined. Also, the execution strategy is not studied in this paper, but techniques such as rolling and receding rolling horizon [19, 20] will be proposed in the future. 


\section{UPC and FFC analysis for the optimal operation in microgrids}

\subsection{UPC mode description}

110 is in charge of regulating the generation output to reach a constant setpoint $P_{D G n}^{*}$. It can be done, for instance, using a conventional PI controller and comparing the power generation measurement with the power generation setpoint. This setpoint can be calculated by an energy management system minimum cost). In this case, any variation of either the demand or the nondispatchable generation within the microgrid will be compensated by the external grid.

\subsection{FFC mode description}

$D G$ is in charge of regulating the power flow (or as we call, the feeder flow) upstrem the generator to reach a constant setpoint $F F_{n}^{*}$. It can be done using a conventional PI controller and comparing the feeder flow measurement with the feeder flow setpoint. Again, this setpoint can be calculated by an EMS generation variation downstream the controlled feeder will be compensated by the dispatchable generators while the power exchange with the external grid will be maintained constant. So, in contrast to the UPC mode, the optimization algorithm for FFC based microgrids should consider the load and downstream non-dispatchable generation variability.

\section{Optimal feeder flow problem formulation}

This section presents the formulation of the optimal operation of a feeder flow controlled microgrid and how it can be solved. The objective is to present the concept of how the operation of feeder flow controlled microgrids can be lation and the proposed algorithm is the same in the case of single period and multi-period, this paper considers a single period. But this formulation could be extended to multi-period optimization problem. Also, for the sake of simplicity just loads, dispatchable generators and non-dispatchable generactive and reactive power setpoints of the UPC generators and the active 


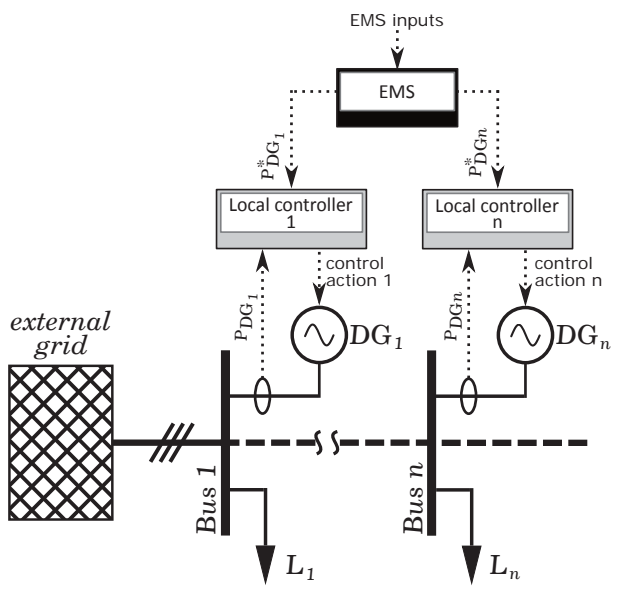

(a) UPC

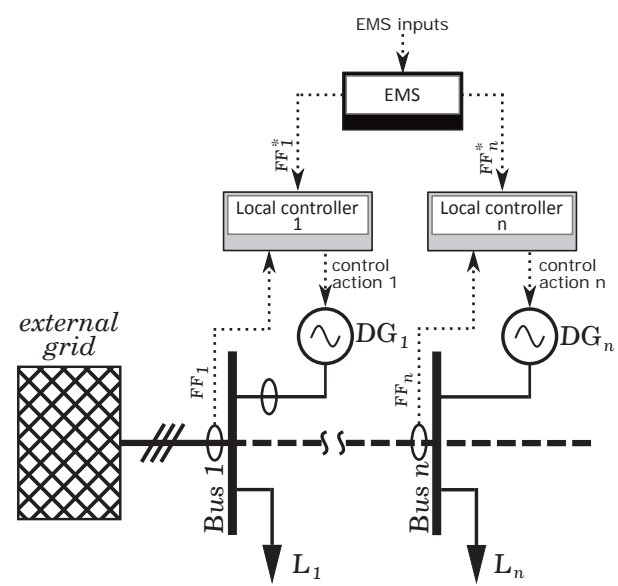

(b) FFC

Figure 1: Power control mode of a DG

and reactive power flow setpoints of the FFC generators. The state of the generator, $y_{n}$, is also a decision variable.

\subsection{Notation}

Tables 1, 2 and 3 show the sets, variables and parameters used in the proposed optimal feeder flow formulation. Note that the voltage and current variables represent square values, avoiding the need of using non-conic constraints [16, 17].

Table 1: Sets definition

\begin{tabular}{ll}
\hline Sets & Description \\
\hline$N$ & Grid nodes $n \in N$ (for slack: $n=1$ ) \\
$S$ & Scenarios \\
$L$ & Lines $(n, m) ; n, m \in N, n=$ up-stream, $m=$ down-stream \\
$u(n)$ & Nodes $m \in N$ that comply both: i) up-stream node $n \in N$ and \\
& ii) directly connected to $n \in N$. i.e. all node $m \in N$ that complies \\
& $(m, n) \in L$ \\
$d(n)$ & Nodes $m \in N$ that comply both: i) down-stream node $n \in N$ and \\
& ii) directly connected to $n \in N$. i.e. all node $m \in N$ that complies \\
& $(n, m) \in L$ \\
$U P C$ & Nodes $n \in N$ with generators under Unit Power Control mode \\
$F F C$ & Nodes $n \in N$ with generators under Feeder Flow Control mode \\
\hline
\end{tabular}


Table 2: Variables definition

\begin{tabular}{|c|c|}
\hline Real variables & Description \\
\hline$P_{n s}^{G}, Q_{n s}^{G}$ & $\begin{array}{l}\text { Active and reactive power generation at node } n \in N \\
\text { and scenario } s \in S\end{array}$ \\
\hline$P_{n m s}, Q_{n m s}$ & $\begin{array}{l}\text { Active and reactive power flow from node } n \in N \text { to } \\
\text { node } m \in N \text { at scenario } s \in S .(n, m) \in L\end{array}$ \\
\hline$P_{n s}^{n e t}, Q_{n s}^{n e t}$ & $\begin{array}{l}\text { Net active and reactive power consumption at node } \\
n \in N \text { at scenario } s \in S\end{array}$ \\
\hline$P_{n}^{G^{*}}, Q_{n}^{G^{*}}$ & $\begin{array}{l}\text { Active and reactive power setpoint at generator } \\
\text { connected at node } n \in U P C\end{array}$ \\
\hline$F F P_{n}^{*}, F F Q_{n}^{*}$ & $\begin{array}{l}\text { Active and reactive power flow setpoint at generator } \\
\text { connected at node } n \in F F C\end{array}$ \\
\hline$\Delta F F P_{n s}, \Delta F F Q_{n s}$ & $\begin{array}{l}\text { Active and reactive power flow deviation at generator } \\
\text { connected at node } n \in F F C \text { at scenario } s \in S\end{array}$ \\
\hline$\Delta C$ & $\begin{array}{l}\text { Auxiliary variable for the convexification of the } \\
\text { objective function }\end{array}$ \\
\hline$\Delta C_{1}$ & Total fixed generation cost \\
\hline$\Delta C_{2}$ & $\begin{array}{l}\text { Total variable penalty cost for feeder flow setpoint } \\
\text { violation }\end{array}$ \\
\hline$\Delta C_{3}$ & Total fix penalty cost for feeder flow setpoint violation \\
\hline Positive variables & Description \\
\hline$l_{n m s}$ & $\begin{array}{l}\text { Square of the current flowing from node } n \in N \text { to } \\
\text { node } m \in N \text { at scenario } s \in S .(n, m) \in L\end{array}$ \\
\hline$\vartheta_{n s}$ & $\begin{array}{l}\text { Square of the voltage of node } n \in N \text { at scenario } \\
s \in S\end{array}$ \\
\hline$D P_{1 s}$ and $D P_{2 s}$ & $\begin{array}{l}\text { Auxiliary variables for linearise the absolute value: } \\
\left|\Delta F F P_{s}\right|=D P_{1 s}+D P_{2 s}, s \in S\end{array}$ \\
\hline$D Q_{1 s}$ and $D Q_{2 s}$ & $\begin{array}{l}\text { Auxiliary variables for linearise the absolute value: } \\
\left|\Delta F F Q_{s}\right|=D Q_{1 s}+D Q_{2 s}, s \in S\end{array}$ \\
\hline Binary variables & Description \\
\hline$y_{n}$ & $\begin{array}{l}\text { State of generator connected at node } n \in N \text {. } \\
\text { Connected }=1 \text {, disconnected }=0\end{array}$ \\
\hline$z p_{s}, z q_{s}$ & $\begin{array}{l}\text { Status of the active and reactive power flow } \\
\text { compliance at slack node and scenario } s \in S \\
0=\text { compliant, } 1=\text { non-compliant }\end{array}$ \\
\hline$z p 1_{n s}, z p 2_{n s}$ & $\begin{array}{l}\text { Auxiliary variables for linearising the condition: if } \\
P_{n}^{G m i n}<P_{n s}^{G}<P_{n}^{G \max } \text { then } \Delta F F P_{n s}=0 \\
n \in F F C, s \in S\end{array}$ \\
\hline$z q 1_{n s}, z q 2_{n s}$ & $\begin{array}{l}\text { Auxiliary variables for linearising the condition: if } \\
Q_{n}^{G m i n}<Q_{n s}^{G}<Q_{n}^{G m a x} \text { then } \Delta F F Q_{n s}=0 \\
n \in F F C, s \in S\end{array}$ \\
\hline$z d p_{s}, z d q_{s}$ & $\begin{array}{l}\text { Auxiliary variables for linearising the absolute } \\
\text { values: }\left|\Delta F F P_{s}\right| \text { and }\left|\Delta F F Q_{s}\right|, s \in S\end{array}$ \\
\hline
\end{tabular}


Table 3: Parameters definition

\begin{tabular}{|c|c|}
\hline Parameters & Description \\
\hline$r_{n m}, x_{n m}$ & Resistance and reactance of the line $(n, m) \in L$ \\
\hline$P_{n s}^{C}, Q_{n s}^{C}$ & $\begin{array}{l}\text { Active and reactive power consumption forecast at node } \\
n \in N \text { at scenario } s \in S\end{array}$ \\
\hline$\vartheta_{\min }, \vartheta_{\max }$ & Minimum and maximum square voltage \\
\hline$\vartheta^{*}$ & Slack voltage \\
\hline$l_{n m}^{\max }$ & Maximum square current flow through line $(n, m) \in L$ \\
\hline$P_{n}^{G \min }, P_{n}^{G \max }$ & $\begin{array}{l}\text { Minimum and maximum active power generation at node } \\
n \in N \text { when the corresponding generator is connected. }\end{array}$ \\
\hline$Q_{n}^{G \min }, Q_{n}^{G \max }$ & $\begin{array}{l}\text { Minimum and maximum reactive power generation at node } \\
n \in N \text { when the corresponding generator is connected. }\end{array}$ \\
\hline$\left.F F P_{n}^{*}\right|_{n=1}$ & External active power flow setpoint at node $n=1$ \\
\hline$\left.F F Q_{n}^{*}\right|_{n=1}$ & External reactive power flow setpoint at node $n=1$ \\
\hline$M_{1}, M_{2}, M_{3}$ & Large values used for modelling integer constraints \\
\hline$C_{n}^{a g}, C_{n}^{b g}, C_{n}^{c g}$ & $\begin{array}{l}\text { Fix, variable and quadratic generation cost parameters } \\
\text { at node } n \in N\end{array}$ \\
\hline$C^{a f f p}, C^{b f f p}$ & $\begin{array}{l}\text { Fix and variable cost parameters of active power flow } \\
\text { non-compliance at node } n=1\end{array}$ \\
\hline$C^{a f f q}, C^{b f f q}$ & $\begin{array}{l}\text { Fix and variable cost parameters of reactive power flow } \\
\text { non-compliance at node } n=1\end{array}$ \\
\hline
\end{tabular}

\subsection{Stochastic formulation}

Errors in demand and PV power forecast can be considered in the optimization problem by using stochastic formulation. In the present paper, a number of scenarios $N_{s}$ is generated. Each scenario consists on determining a estimated load demand and PV generation at each node. These scenarios are generated from the forecasted values and from Gaussian error probability distributions. Then, the decision variables, which are the generators setpoints, are unique for all scenarios (see section 3.4). In contrast, the power flow is computed for each scenario considering the unique setpoints as shown in section 3.3. On the other hand, the objective function is the sum of the objective function of each scenario. Note that those most-probable scenarios will contribute more to the final cost of the objective function, as they will appear more times: 1 . Also note that the voltage, current and power generation limits are ensured for all the scenarios.

\footnotetext{
${ }^{1}$ This method is commonly known as Sample-Average Approximation (SAA)
} 


\subsection{Power flow equations}

First of all, and for the sake of comprehension, the scheme of the DistFlow formulation model [21, for optimal power flow formulation, is introduced in Figure 2. In this Figure, the sub-index corresponding to each scenario $s \in S$ is omitted. Also, the external active and reactive power flow setpoints are depicted. The net consumption $\left(P_{n}^{n e t}\right.$ and $\left.Q_{n}^{\text {net }}\right)$ is drawn as a load, but note that generators can also be installed. Generation is considered as a negative net consumption.

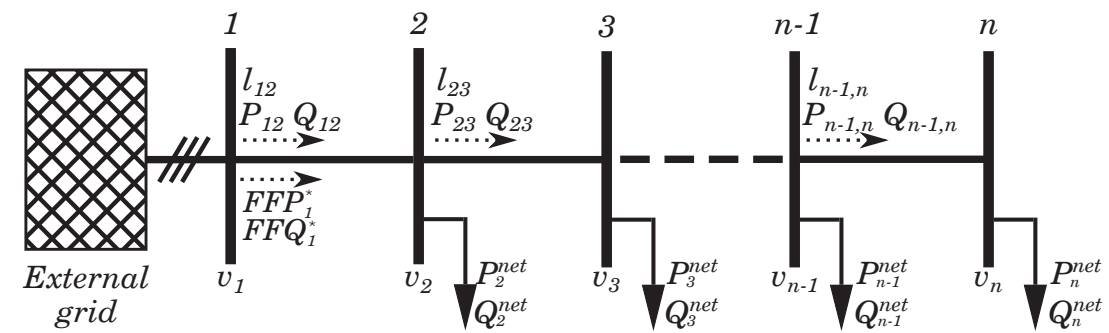

Figure 2: Scheme of the DistFlow model

The active and reactive power balance constraints at the slack node are expressed as (1) and (2), respectively. The power balance at the rest of the nodes is expressed as (3) and (4), respectively (the net consumption is equal to the input power minus the output power).

$$
\begin{gathered}
P_{n s}^{G}=\sum_{m \in d(n)} P_{n m s} \forall s \in S ; n=1 \\
Q_{n s}^{G}=\sum_{m \in d(n)} Q_{n m s} \forall s \in S ; n=1 \\
P_{n s}^{n e t}=\sum_{m \in u(n)}\left(P_{m n s}-r_{m n} \cdot l_{m n s}\right)-\sum_{m \in d(n)} P_{n m s} \forall s \in S ; \forall n \in N \backslash\{1\} \\
Q_{n s}^{n e t}=\sum_{m \in u(n)}\left(Q_{m n s}-x_{m n} \cdot l_{m n s}\right)-\sum_{m \in d(n)} Q_{n m s} \forall s \in S ; \forall n \in N \backslash\{1\}
\end{gathered}
$$

The net power consumption is calculated as (5) and (6), respectively. It represents the forecasted load minus the generated power (which can be forecasted in case of non-dispatchable generation or a decision variable in case of dispatchable generation). 


$$
\begin{aligned}
& P_{n s}^{n e t}=P_{n s}^{C}-P_{n s}^{G} \forall n \in N ; \forall s \in S \\
& Q_{n s}^{n e t}=Q_{n s}^{C}-Q_{n s}^{G} \forall n \in N ; \forall s \in S
\end{aligned}
$$

The voltage drops can be expressed as (7). Then, the voltages must be constrained between upper and lower limits as shown in (8). The slack voltage, i.e. the point of the microgrid interconnection, is fixed according to constraint (9).

$$
\begin{gathered}
\vartheta_{m s}=\vartheta_{n s}-2\left(r_{n m} P_{n m s}+x_{n m} Q_{n m s}\right)+ \\
l_{n m s}\left(r_{n m s}^{2}+x_{n m s}^{2}\right) \quad \forall n \in N ; \forall m \in d(n) ; \forall s \in S \\
\vartheta_{m i n} \leq \vartheta_{n s} \leq \vartheta_{\max } \forall n \in N ; \forall s \in S \\
\vartheta_{n s}=\vartheta^{*} \forall s \in S ; n=1
\end{gathered}
$$

The square of the currents flowing through the lines are also constrained, as shown in 10 .

$$
l_{n m s} \leq l_{n m}^{\max } \forall n \in N ; \forall m \in d(n) ; \forall s \in S
$$

The generation limits are expressed as (11)-(14), being $y_{n}$ the connection/disconnection binary variable.

$$
\begin{aligned}
& P_{n}^{G \min } \cdot y_{n} \leq P_{n s}^{G} \forall n \in N ; s \in S \\
& P_{n s}^{G} \leq P_{n}^{G \max } \cdot y_{n} \forall n \in N ; s \in S \\
& Q_{n}^{G \min } \cdot y_{n} \leq Q_{n s}^{G} \forall n \in N ; s \in S \\
& Q_{n s}^{G} \leq Q_{n}^{G \max } \cdot y_{n} \forall n \in N ; s \in S
\end{aligned}
$$

The current can be calculated as (15). This constraint is clearly non linear and non-convex. This leads to the need of using non linear programming (NLP) solvers, which rarely find the global optimum. To solve this issue, this constraint is relaxed as (16), becoming a second order cone constraint.

$$
\begin{gathered}
l_{n m s}=\frac{P_{n m s}^{2}+Q_{n m s}^{2}}{\vartheta_{n s}} \forall n \in N ; \forall m \in d(n) ; \forall s \in S \\
l_{n m s} \vartheta_{n s} \geq P_{n m s}^{2}+Q_{n m s}^{2} \forall n \in N ; \forall m \in d(n) ; \forall s \in S
\end{gathered}
$$




\subsection{UPC and FFC constraints}

Generators of the microgrid can operate under FFC or UPC modes. For generators operating under UPC mode, the active and reactive power generation must be the same for all scenarios. This is expressed with constraints (17) and (18).

$$
\begin{aligned}
& P_{n s}^{G}=P_{n}^{G^{*}} \forall n \in U P C ; \forall s \in S \\
& Q_{n s}^{G}=Q_{n}^{G^{*}} \forall n \in U P C ; \forall s \in S
\end{aligned}
$$

For those generators under FFC mode, the power flow upstream their associated bus must be equal to the setpoint for all scenarios. This can be expressed by the constraints $(19)$ and $(20)$.

$$
\begin{aligned}
& F F P_{n}^{*}=\sum_{m \in d(n)}\left(P_{n m s}\right)+P_{n s}^{n e t} \forall n \in F F C ; \forall s \in S \\
& F F Q_{n}^{*}=\sum_{m \in d(n)}\left(Q_{n m s}\right)+Q_{n s}^{n e t} \forall n \in F F C ; \forall s \in S
\end{aligned}
$$

The above equations enforce the power flow through the controlled lines to be constant for all scenarios. This can lead to an infeasible solution. If the downstream loads variations between scenarios are large enough, the active power generation required from the feeder flow controlled generators can be greater than their maximum power (constraints $\sqrt{12}$ ) and (14) ) or lower than their minimum power (constraints $(11)$ and $(13)$ ). Note that if 205 multiple FFC generators are connected, the upstream FFC generators can mitigate the limits of the downstream FFC generators [10], resulting in a feasible problem. This motivates to find an alternative formulation that can consider this effect. Without losing the convexity of the OPF problem, the feeder flow constraints are rewritten as (21)-25) for the active power and as (26)-30 for the reactive power. 


$$
\begin{gathered}
F F P_{n}^{*}+\Delta F F P_{n s}=\sum_{m \in d(n)}\left(P_{n m s}\right)+P_{n s}^{n e t} \forall n \in F F C ; \forall s \in S \\
P_{n s}^{G}-y_{n} P_{n}^{G m i n} \leq P_{n}^{G m a x} \cdot z p 1_{n s} \forall s \in S ; \forall n \in F F C \\
y_{n} P_{n}^{G m a x}-P_{n s}^{G} \leq P_{n}^{G m a x} \cdot z p 2_{n s} \forall s \in S ; \forall n \in F F C \\
\Delta F F P_{n s} \leq M_{1}\left(2-z p 1_{n s}-z p 2_{n s}\right) \forall s \in S ; \forall n \in F F C \\
\Delta F F P_{n s} \geq M_{1}\left(z p 1_{n s}+z p 2_{n s}-2\right) \forall s \in S ; \forall n \in F F C \\
F F Q_{n}^{*}+\Delta F F Q_{n s}=\sum_{m \in d(n)}\left(Q_{n m s}\right)+Q_{n s}^{n e t} \forall n \in F F C ; \forall s \in S \\
Q_{n s}^{G}-y_{n} Q_{n}^{G m i n} \leq Q_{n}^{G m i n} \cdot z q 1_{n s} \forall s \in S ; \forall n \in F F C \\
y_{n} Q_{n}^{G m a x}-Q_{n s}^{G} \leq Q_{n}^{G m a x} \cdot z q 2_{n s} \forall s \in S ; \forall n \in F F C \\
\Delta F F Q_{n s} \leq M_{1}\left(2-z q 1_{n s}-z q 2_{n s}\right) \forall s \in S ; \forall n \in F F C \\
\Delta F F Q_{n s} \geq M_{1}\left(z q 1_{n s}+z q 2_{n s}-2\right) \forall s \in S ; \forall n \in F F C
\end{gathered}
$$

If the constraints for the FFC of the active power (21)-(25) are considered . Equation (21) reflects that all scenarios have the same active power flow setpoint $\left(F F P_{n}^{*}\right)$, but they can present different deviations $\left(\triangle F F P_{n s}\right)$. Equations (22) and (23) say that i) if $P_{n s}^{G}$ is strictly greater than $P_{n}^{G m i n}$, then $215 z p 1_{n s}$ is equal to 1 and $\left.i i\right)$ if $P_{n s}^{G}$ is strictly lower than $P_{n}^{G m a x}$, then $z p 2_{n s}$ is equal to 1 . So, if the power generation $P_{n s}^{G}$ is not at its limits, then the condition (31) is fulfilled. If $M_{1}$ is large enough, equation (24) says that if the condition (31) is fulfilled, then $\triangle F F P_{n s}$ is lower or equal to 0 . Otherwise, $\Delta F F P_{n s}$ can take any value. The same way, equation (25) says that if the condition (31) is fulfilled, then $\triangle F F P_{n s}$ is greater or equal to 0 . Otherwise, $\triangle F F P_{n s}$ can take any value. Hence, if condition (31) is fulfilled, then $\triangle F F P_{n s}=0$ which means that the active power flow setpoint is fulfilled by the scenario $s$. Otherwise, if the condition (31) is not fulfilled, then $\triangle F F P_{n s}$ can take any value. Note that (31) is used for explaining the FFC constraints and is not included in the optimization problem.

$$
z p 1_{n s}+z p 2_{n s}=2
$$

Reactive power feeder flow constraints (26)-(30) behave in the same manner. Note that these equations are linear integer constraints which suits well with the MISOCP formulation. 


\subsection{Objective function}

230

As the active and reactive power exchanges between the grid and the microgrid will be determined by an external agent, the most interesting objective function is the operation cost minimization.

The operation cost can be written as $(32)$. The cost function considers quadratic generation costs plus a penalty for feeder flow setpoint deviations.

This penalty is linear with the absolute value of the corresponding deviation.

$$
\begin{gathered}
f=\sum_{s \in S}\left[\sum_{n \in N}\left(C_{n}^{a g} \cdot y_{n}+C_{n}^{b g} \cdot P_{n s}^{G}+C_{n}^{c g} \cdot P_{n s}^{G^{2}}\right)\right]+ \\
\sum_{s \in S}\left[C^{a f f p} \cdot z p_{s}+C^{b f f p} \cdot\left|\Delta F F P_{s}\right|\right]+ \\
\sum_{s \in S}\left[C^{a f f q} \cdot z q_{s}+C^{b f f q} \cdot\left|\Delta F F Q_{s}\right|\right]
\end{gathered}
$$

where $\left|\Delta F F P_{s}\right|=\left|F F P^{*}-F F P_{s}\right|$ and $\left|\Delta F F Q_{s}\right|=\left|F F Q^{*}-F F Q_{s}\right|$ are the feeder flow setpoint deviations at the slack node.

The objective function contains absolute values and binary variables. This function is reformulated to obtain a convex quadratic function. The above cost function is rewritten as (33) with the additional constraints (34)(46).

$$
f=\sum_{s \in S}\left[\sum_{n \in N}\left(C_{n}^{b g} \cdot P_{n s}^{G}+C_{n}^{c g} \cdot P_{n s}^{G^{2}}\right)\right]+\Delta C
$$

The total activation cost of generators is expressed as (34).

$$
\Delta C_{1}=\sum_{s \in S}\left[\sum_{n \in N}\left(C_{n}^{a g} \cdot y_{n}\right)\right]
$$

The variable cost of feeder flow setpoint deviation is linearised as follows. Equation (35) represents the cost while constraints (36)- 42 are for linearising the absolute values. 


$$
\begin{gathered}
\Delta C_{2}=\sum_{s \in S}\left[\left(D P_{1 s}+D P_{2 s}\right) \cdot C^{b f f q}+\left(D Q_{1 s}+D Q_{2 s}\right) \cdot C^{b f f q}\right] \\
P_{n s}^{G}-F F P^{*}=D P_{1 s}-D P_{2 s} \forall s \in S ; n=1 \\
D P_{1 s} \leq M_{2} \cdot z d p_{s} \forall s \in S \\
D P_{2 s} \leq M_{2} \cdot\left(1-z d p_{s}\right) \forall s \in S \\
Q_{n s}^{G}-F F Q^{*}=D Q_{1 s}-D Q_{2 s} \forall s \in S ; n=1 \\
D Q_{1 s} \leq M_{2} \cdot z d q_{s} \forall s \in S \\
D Q_{2 s} \leq M_{2} \cdot\left(1-z d q_{s}\right) \forall s \in S \\
D P_{1 s}, D P_{2 s}, D Q_{1 s}, D Q_{2 s} \geq 0
\end{gathered}
$$

Note that $\left|\Delta F F P_{s}\right|=D P_{1 s}+D P_{2 s}$ and $\left|\Delta F F Q_{s}\right|=D Q_{1 s}+D Q_{2 s}$.

The fixed cost of feeder flow setpoint deviation is expressed as (43). Constraints (44) and (45) determine if the scenario $s$ fulfils (or not) the external setpoints through the variables $z p_{s}$ and $z q_{s}$.

$$
\begin{gathered}
\Delta C_{3}=\sum_{s \in S}\left[z p_{s} \cdot C^{a f f p}+z q_{s} \cdot C^{a f f q}\right] \\
D P_{1 s}+D P_{2 s} \leq M_{3} \cdot z p_{s} \forall s \in S \\
D Q_{1 s}+D Q_{2 s} \leq M_{3} \cdot z q_{s} \forall s \in S
\end{gathered}
$$

Then, as the optimal feeder flow is a minimization problem, the cost $\Delta C$ can be expressed as (46). Note that due to the minimization nature of the problem, the solver will always find the solution $\Delta C=\Delta C_{1}+\Delta C_{2}+\Delta C_{3}$.

$$
\Delta C_{1}+\Delta C_{2}+\Delta C_{3} \leq \Delta C
$$

\subsection{Optimal feeder flow problem}

In [16], the second order cone relaxation for the optimal power flow problem is studied. The authors prove that the relaxed problem is exact under the following conditions?

\footnotetext{
${ }^{2}$ Refer to [16] for more details. These conditions have been proven for the SOCP formulation. For MISOCP, the case of this paper, these conditions work but there is not a formal mathematical proof yet. For example, it has been successfully implemented in [22.
} 
A1) The network graph is connected.

A2) The objective function is convex.

A3) The objective function is strictly increasing in currents $(l)$, non increasing in load, and independent of the apparent power.

A4) The original optimal power flow problem is feasible.

Being exact means that every optimal solution of the relaxed problem satisfies equation (15). Hence, as the optimal solution is feasible for the non-relaxed problem, then it is the global optimum.

The optimal feeder flow problem is defined as following and denoted in this paper as OFFP:

$$
[M I N] f
$$

Subject to (1)-(14), (16)-(18), (21)-(30), (34)-(46).

Condition A1 is expected to occur always. Condition A4 is a basic requirement to find a solution and will depend on the grid design. Hence, Only conditions A2 and A3 have to be checked. In the proposed problem, the objective function is convex (A2). On the other hand, condition A3 may not be always satisfied. Note that we include additional penalty costs terms and constraints. This motivates to find an alternative solution for solving the OFFP.

275 The proposed solution consists on applying a penalty to the objective function so that the global optimum is forced to be at the surface of the cone represented by the relaxed constraint (16), i.e. forcing the relaxed constraint to be active. If the relaxation does not hold, it means that the objective function can be improved if the power losses are artificially increased, leading to a non-meaningful solution. This could occur for example when the feeder flow setpoint is not fulfilled but increasing the power losses it can be fulfilled diminishing the corresponding penalties. Hence, the solution consists on adding the currents to the objective function with a penalty weight $w$. The objective function is redefined as (47).

$$
f^{\prime}=f+w \cdot \sum_{s \in S} \sum_{n \in N} \sum_{m \in d(n)} l_{n m s}
$$


Then, the corresponding optimal feeder flow problem (denoted as OFFP$\mathbf{w})$ is defined as:

\section{$[M I N] f^{\prime}$}

Subject to (1)-(14), (16)-(18), (21)-(30), (34)- 46).

The main difficulty is still to determine the value of $w$. If the relaxation does not hold, it means that increasing artificial losses still improves the objective function (the penalty $w$ is too low). On the other hand, if the relaxation holds but $w$ is too high, the problem will be solved to obtain the minimum currents and not the minimum costs represented by $f$. To find the appropriate weight, i.e. the minimum $w$ that makes the relaxation hold, the algorithm 1 is developed.

The algorithm begins with no penalty. So, the OFFP-w is equivalent to the OFFP. If relaxation does not hold, then high and low bounds of the penalty weight are found. The initial range is set between 0 and the value which would make the penalty contribution to be equal to 10 times the objective function. The next iteration will hold thanks to this large penalty weight. Then at each iteration, $w$ is set at the middle of the range [low-high]. If the problem is solved and the relaxation does not hold, $w$ is too small and the low value is updated. Otherwise, the high value is updated. Note that at each iteration this range is reduced by half. The algorithm terminates when $w$ cannot vary more that $5 \%$ or when the objective function is smaller than the lower bound found at the first optimization $+1 c €$.

The OFFP and the OFFP-w problems are MISOCP optimization problems. These can be efficiently solved by the CPLEX solver using the Branch and Cut method [17]. In this paper the CPLEX solver is used under the GAMS environment.

\section{Case study}

The test system is based on the IEEE 33-Bus distribution system [23] as shown in Figure 3. This is a well-known test network that has been previously used for studying the optimal operation of microgrids [24]. The load forecasts for the lines 6-18 and 26-33 are increased 1.5 times. In addition, 6 PV generators and 8 dispatchable generators have been included. According to [13], a multi-FFC configuration suits better for microgrids without dominant generators. On the other hand, different configurations have been discussed 


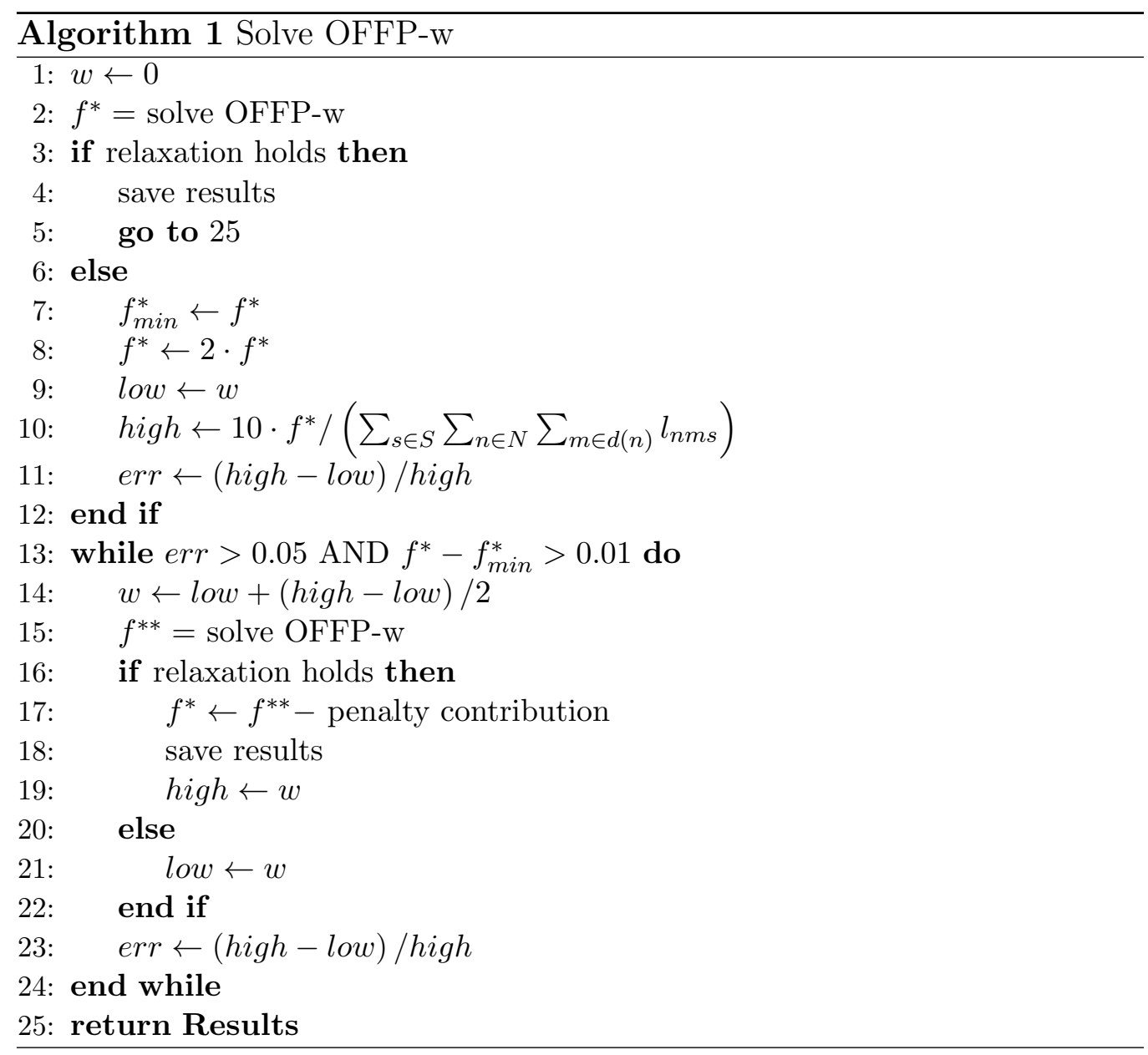


in [10], revealing that if a single FFC generator is controlling the microgrid, then it should be dominant. So, in [10] the multiple FFC generators are also proposed as a more suitable option. Another option could be all generators operating under FFC mode. But following the schemes of [10] and [13], the presented test case considers $3 \mathrm{FFC}$ generators and several UPC generators. Generators identified as 2, 6 and 26 are operated under FFC mode, while the rest of dispatchable generators operate under UPC mode. Note that as generator 2 operates as a FFC unit controlling the power exchange with the utility grid, the microgrid can be considered as a FFC microgrid. The total power consumption is around 3.7 MW, while the installed power generation is $5 \mathrm{MW}$. The load and PV forecast errors (standard deviation) are set to realistic values. For PV forecast it can be expected a root mean square error between $10 \%$ to $15 \%$ [25, 26]. In this paper, a PV forecast error of $15 \%$ is assumed. On the other hand, the load forecast errors can be assumed to be between $5 \%$ and $12 \%$ in microgrid buildings [27]. In this paper, a $10 \%$ of load forecast errors is considered. These errors follow a Gaussian distribution.

It is considered that PV generators operate under maximum power point control mode. Hence, their active power generation is forced to be equal to the corresponding prediction.

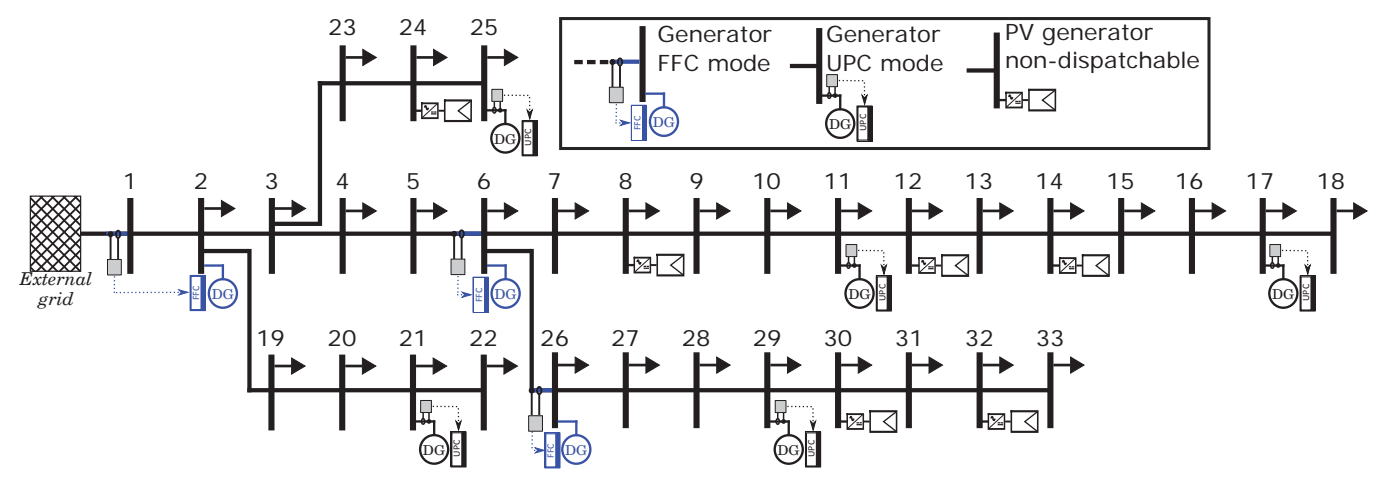

Figure 3: Scheme of IEEE 33-Bus distribution system

\section{Results}

This section presents the results of the proposed optimization problem. First, the proposed algorithm for solving the OFFP is validated showing it converges, finding a feasible optimum solution. Then the proposed stochastic 
formulation is compared to a non-stochastic formulation, showing it finds better solutions for the feeder flow controlled microgrids.

\subsection{Convergence}

First, the forecasted scenario (active and reactive power for loads and active power for PV units) is used to generate $n s$ random scenarios. These $n s$ scenarios are used for testing the proposed optimization algorithm. A total of $n s=10$ scenarios are considered (Figure 4) as it allows finding the optimal solution within a reasonable computation time.

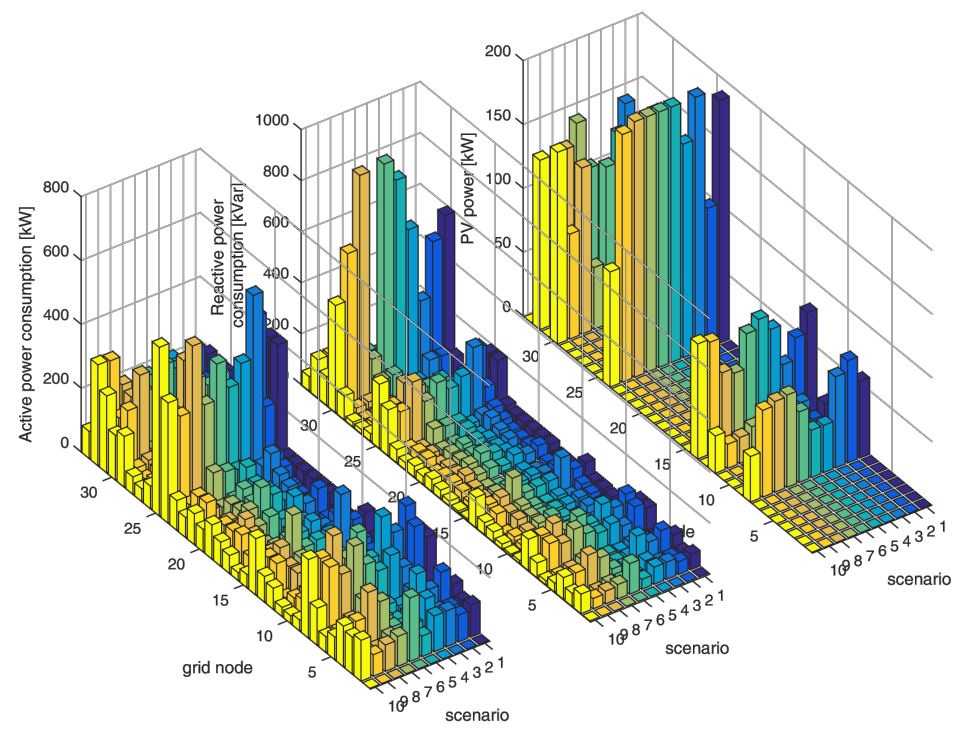

Figure 4: Scenarios used for the optimization problem

To test the solution algorithm convergence, the feeder flow setpoints are fixed to $\left(\left.F F P_{n}^{*}\right|_{n=1},\left.F F Q_{n}^{*}\right|_{n=1}\right)=(1350 \mathrm{~kW}, 900 \mathrm{kvar})$. Figure 5 shows the convergence of the algorithm. In this case, the OFFP relaxation has not hold, but has provided a lower bound of the objective function of $5569 €$ (dashed black line). As a result the low and high values are calculated and $w$ is updated at each iteration. As it can be observed, when the relaxation holds, the high value is decreased. Otherwise, the low value is increased. The algorithm requires 12 iterations, terminating because err $<0.05$. A total cost of $5607 €$ has been obtained, being only $1 \%$ higher than the lower bound found. Hence, it can be considered as a good solution. The proposed algorithm can converge even faster, without degrading the solution in excess, 
if the termination criteria is modified to be less restrictive. For example, iteration 6 has found a solution of $5616 €$, being just $9 €$ higher than the solution found.

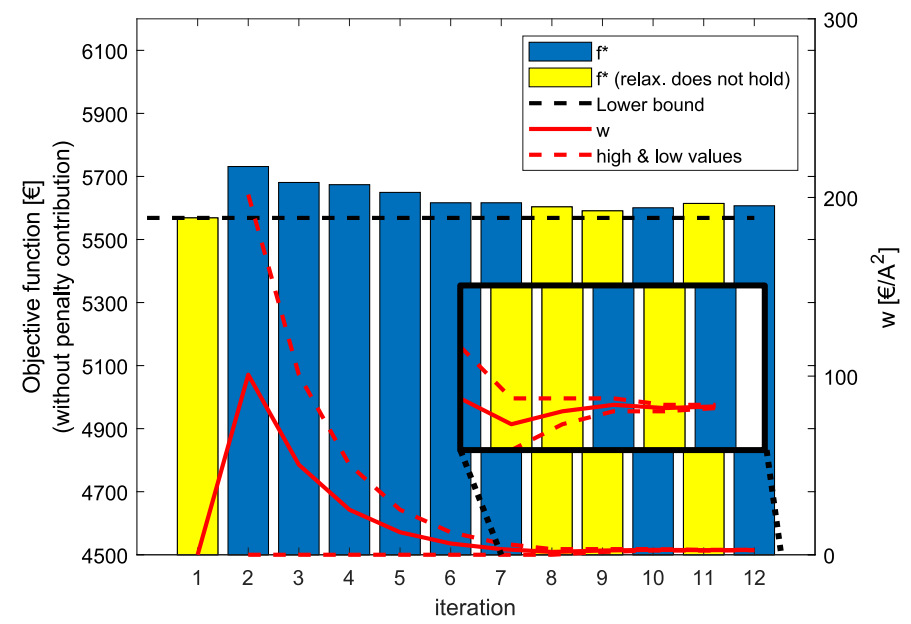

Figure 5: Convergence process of the proposed algorithm

Once the convergence process has been shown, the performance of the algorithm is further analysed in Figure 6. The top plot in Figure 6 shows a sensibility analysis of the relaxation gap, $\epsilon_{r}$, in front of the weight $w$ in blue markers and the $w$ values at each iteration in black circles. The relaxation gap has been defined as (48). This gap measures how far is the relaxed solution from the non-relaxed equality constraint (15), i.e. the OFFP-w holds only if $\epsilon_{r}=0$, otherwise, the optimal solution of the relaxed problem 370 has no physical meaning. The bottom plot shows a sensibility analysis of the objective function in front of $w$. The objective function of the original OFFP is shown in blue, and the added penalty in the modified OFFP-w is shown in red.

$$
\epsilon_{r}=\sum_{s \in S} \sum_{n \in N} \sum_{m \in d(n)}\left[l_{n m s} \vartheta_{n s}-\left(P_{n m s}^{2}+Q_{n m s}^{2}\right)\right]
$$

The top plot in Figure 6 shows how for $w$ greater than 5.2, the OFFP-w 375 holds, i.e. $\epsilon_{r}=0$. As the relaxation has not hold (iteration 1), an initial large $w$ is calculated (iterarion 2), and then, the proposed algorithm searches 
for the minimum $w$ that makes the relaxation holds. According to the bottom plot, which shows that the real objective function increases with $w$, the algorithm has found the best possible solution.
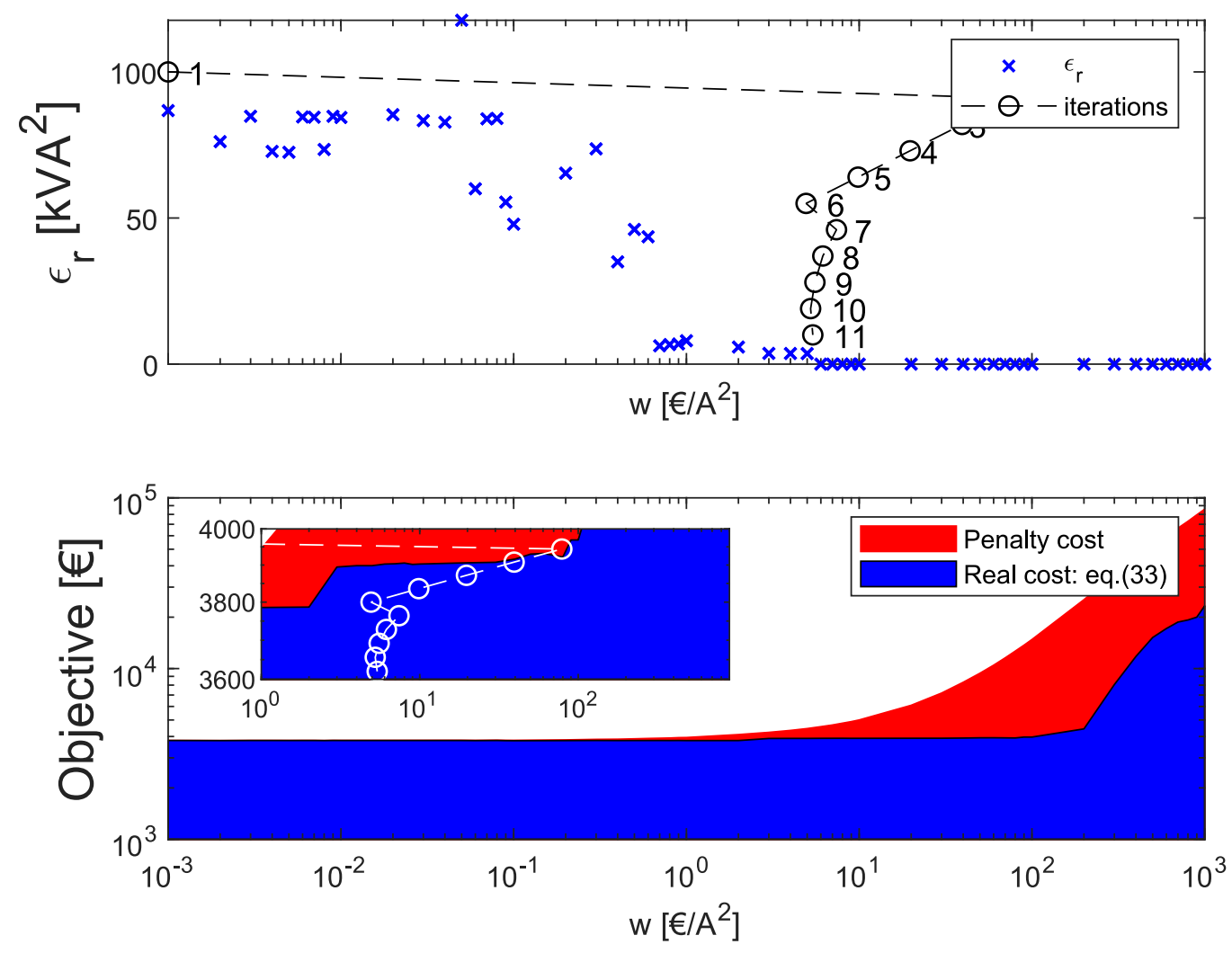

Figure 6: Algorithm evolution. Analysis of the relaxation gap and the objective function

\subsection{Solution comparison}

In [17] an algorithm for solving the relaxed SOCP-OPF was proposed (here it will be summarized but refer to the source for more details). It consists on minimizing only the part of the objective function that complies with condition A3. For this purpose, the new objective function, $f^{\prime \prime}$, only considers the variable generation cost (49).

$$
f^{\prime \prime}=\sum_{s \in S}\left[\sum_{n \in N}\left(C_{n}^{b g} \cdot P_{n s}^{G}+C_{n}^{c g} \cdot P_{n s}^{G^{2}}\right)\right]
$$


Then, constraint 46 is replaced by 50 . Here, $\Delta C^{\prime}$ is a parameter which is modified at each iteration.

$$
\Delta C_{1}+\Delta C_{2}+\Delta C_{3} \leq \Delta C^{\prime}
$$

Then, the corresponding optimal feeder flow problem (denoted as OFFPb) is defined as:

$$
[M I N] f^{\prime \prime}
$$

Subject to (1)-(14), (16)-(18), (21)-(30), (34)-(45), (50).

Then the Algorithm 2 is used for solving the optimization problem.

The convergence and the solution of this algorithm is shown in Figure 7 . The first iteration corresponds to the OFFP solution, which is the same as in the previous algorithm and the relaxation does not hold. Then, the next iterations use the OFFP-b. At the beginning, from iterations 2 to $5, \Delta C^{\prime}$ is large enough so that the relaxation holds. During these iterations, the real objective function (blue bars) is reduced because of the reduction of the $\Delta C^{\prime}$ parameter in (50) (red line). When $\Delta C^{\prime}$ is too low, the relaxation does not hold or the problem becomes infeasible, and then $\Delta C^{\prime}$ is increased (and when relaxation holds again, it decreases). At the end, the real objective function is $6062 €$, being $455 €$ higher than the the one found by the proposed Algorithm 1 .

Considering that random scenarios are generated as input parameters of the optimization problem, the solution comparison should be made based on a set of optimization results. The comparison between the two optimization algorithms can be observed in Figure 8. In this case, 150 optimizations have been launched for both algorithms. The probability density function of the optimization objective have been obtained using the Kernel distribution. It can be observed that the proposed algorithm achieves lower average cost. 


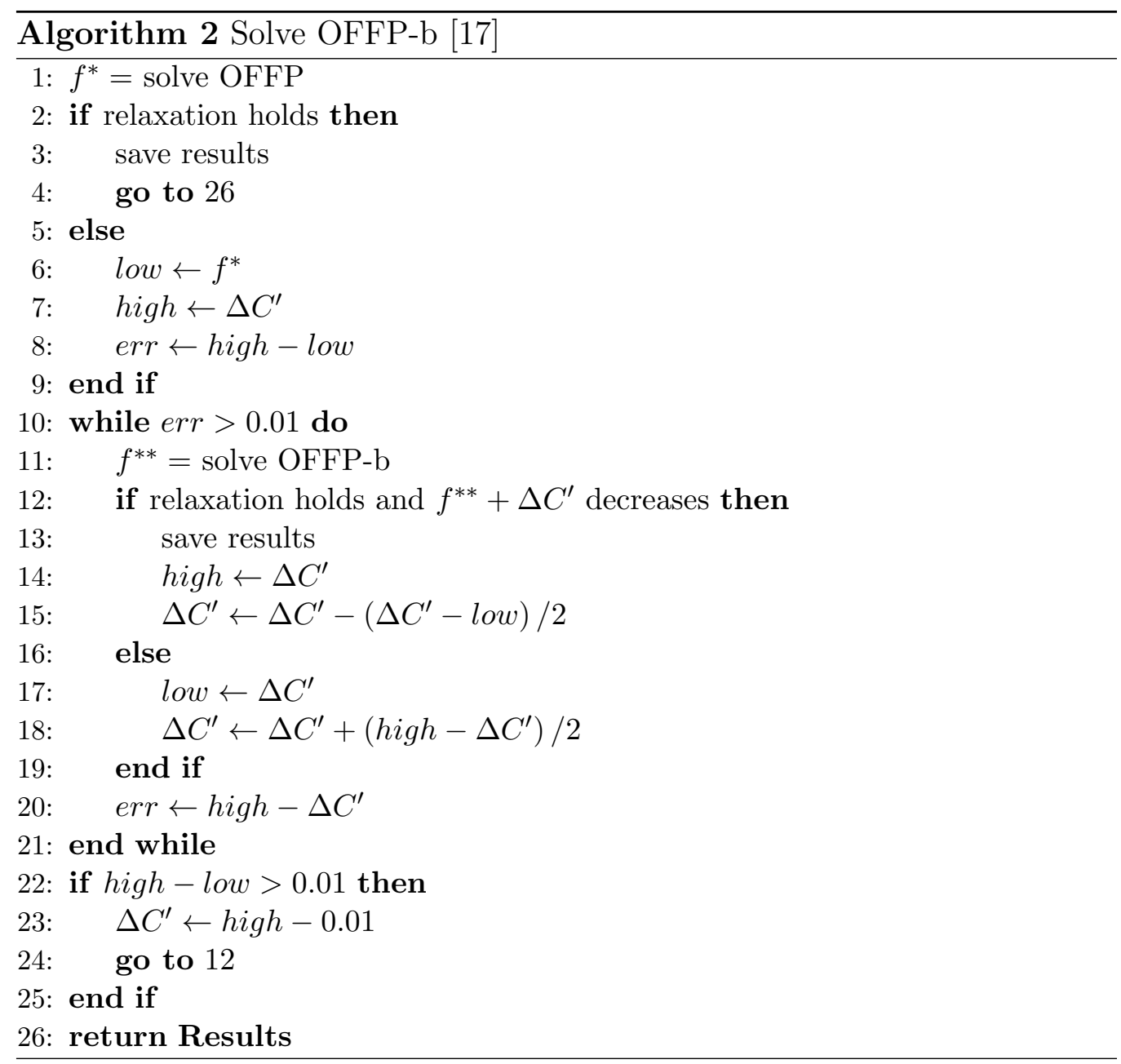




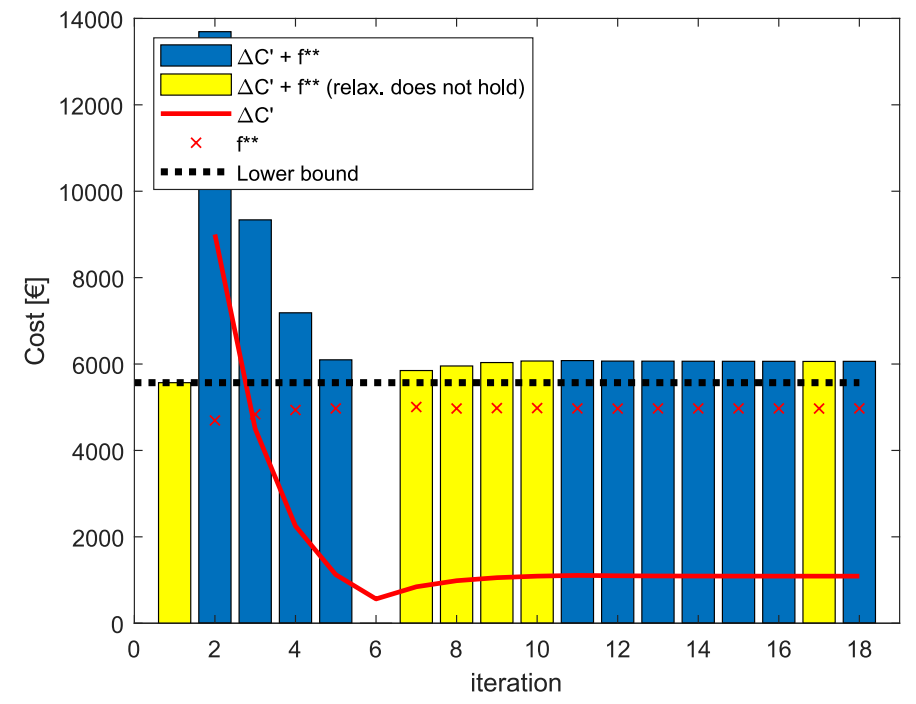

Figure 7: Convergence process of the algorithm proposed in [17]

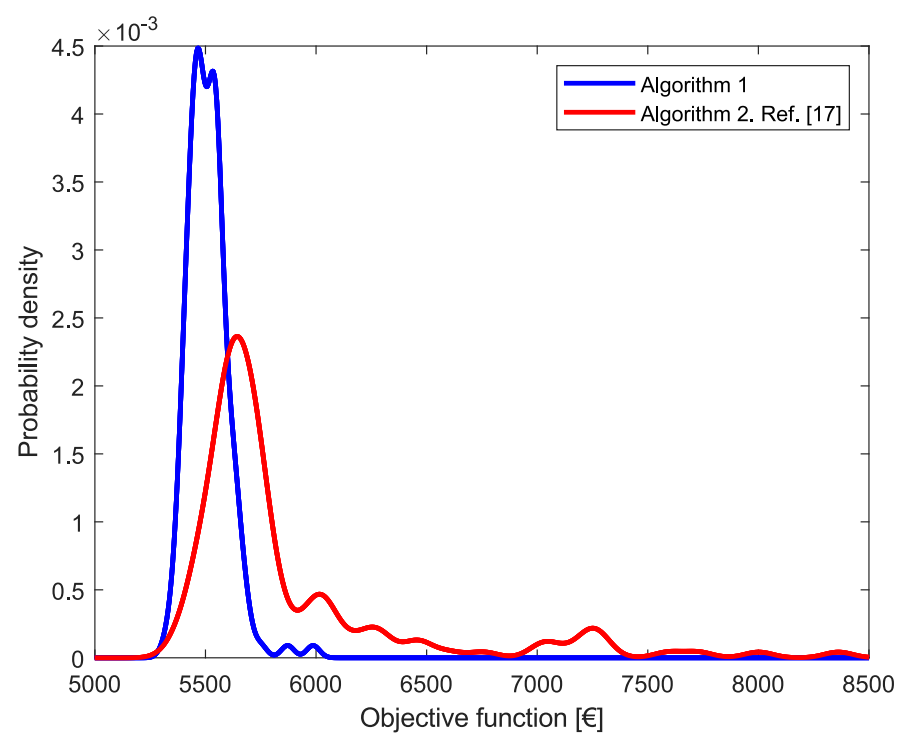

Figure 8: Comparison of the solutions obtained from Algorithm 1 and 2 (150 optimizations). Probability density function of the objective function (Kernel distribution)

\subsection{Computational requirements}

One of the main drawbacks of stochastic optimization is the computational time. As more scenarios are considered, the uncertainty is better 
modelled but the computational time increases. So, it should be analysed the computational cost for selecting the number of scenarios. Figure 9 shows the probability density function of the computational time, which has been obtained using the Kernel distribution after launching 150 optimizations. The computational cost for 1 scenario will rarely exceed $10 \mathrm{~s}$. On the contrary, for 5, 10 and 15 scenarios, the maximum expected computational cost will be around 20, 65 and 100 s respectively. Considering the required optimization time is greatly affected by the number of scenarios, we choose a total of 10 scenarios.

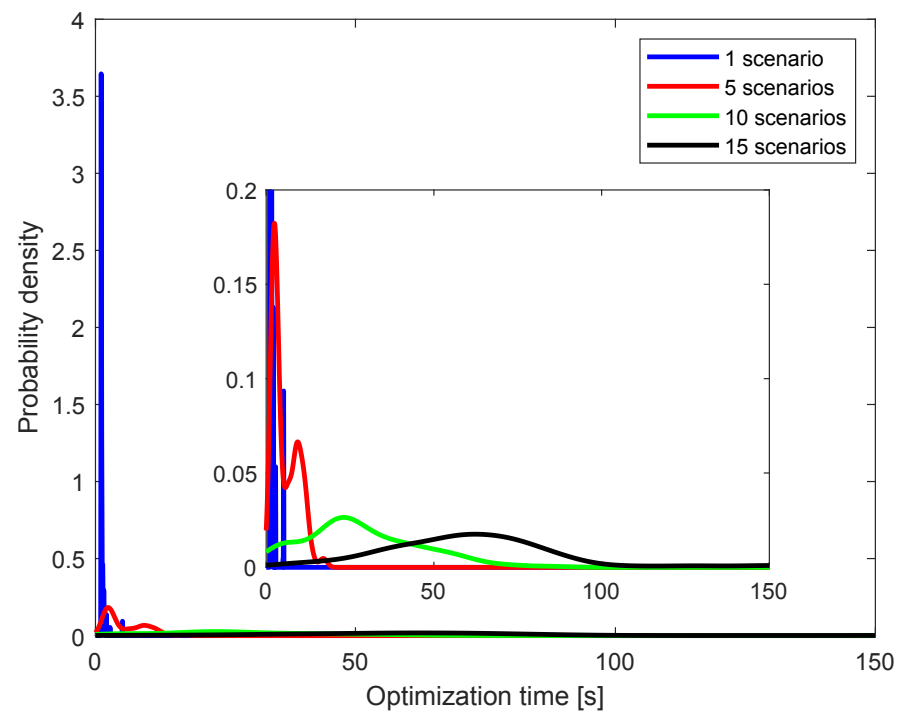

Figure 9: Probability density function of the optimization time for different number of scenarios (Kernel distribution)

\subsection{Operation performance}

This section presents the benefits of the presented OFFP formulation considering uncertainty (stochastic formulation). Until now, the optimization problem has been analysed in terms of convergence and computational time. Also, the objective function has been compared with the resolution method proposed in [17]. As a limited number of scenarios is considered at each optimization, the real performance after applying the output setpoints (feeder flow setpoints for FFC generators and active and reactive power setpoints for UPC generators) should be further analysed. For this purpose, a Monte Carlo procedure has been done as follows: 
1) Define a number of scenarios for the stochastic optimization. We choose 1 scenario (no stochastic) and 10 scenario (stochastic case).

2) Launch the optimization

3) Apply the optimal setpoints to a number random scenarios and execute the power flow solution

4) Save results (objective function and feeder flow compliance at the slack)

5) Go to 2) and repeat

Note that due to the random nature of optimization parameters (forecasted scenarios), analysing the results performance of one single optimization is not adequate. In total, 100 optimization have been performed and 3000 random scenarios have been evaluated to obtain the results shown in Figure 10. It is shown the probability distributions of the optimization objective in function of the number of scenarios used in the optimization stage and the total feeder flow setpoint deviation. It can be observed that the objective function can be reduced by using the stochastic formulation (Figure 10(a). This reduction is related to the better compliance of the external feeder flow setpoint as observed in Figure10(b). The FFC generators has higher operation cost. Nevertheless, the stochastic optimization increases their power generation in order to provide them the capability to decrease the output power achieving a better feeder flow control performance. In this case, the stochastic optimization has been capable to reduce the total cost by $2.5 \%$, and to reduce the number of feeder flow non-compliance cases by $59 \%$ (Figure 10(b) only shows the non-compliant cases over the 3000 cases). penalty costs increase.

A second scenario considers analysing the voltage limits fulfilment. For this purpose, higher feeder flow setpoints are considered, leading to higher voltage drops. In addition, the voltage at the slack node is $0.98 \mathrm{p} . \mathrm{u}$. This leads to a challenging situation where the voltage could exceed the limits due to the larger voltage drops, specially downstream node 6 . Table 4 shows the optimal outputs. When uncertainty is not considered, generator 25 is switched off, while the FFC generator 26 is turned on. In addition, the feeder flow setpoint at generator 26 is small enough to ensure it operates at its maximum power. This permits to diminish the voltage drop from node 1 


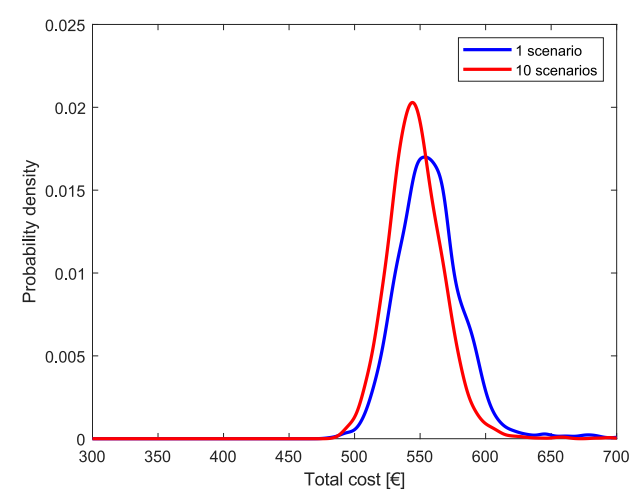

(a) Objective funciton

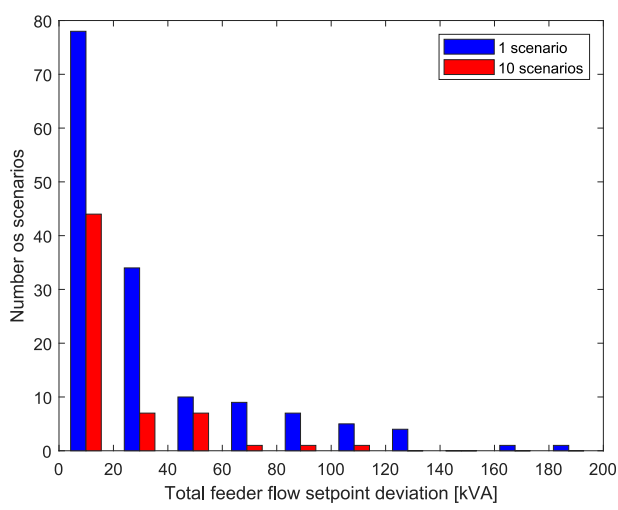

(b) Feeder flow compliance

Figure 10: Optimization problem performance. Comparison between using single forecasted scenario and 10 random scenarios for the optimization process

to node 26 improving the voltage profile. Nevertheless, deviations from the forecast can produce voltage violations as shown in Figure 11 in blue lines, which represents the corresponding simulation for 25 random scenarios. On the other hand, when considering uncertainty, these voltage violations are mitigated thanks to the connection of the FFC generator 6, as shown in red in Figure 11.

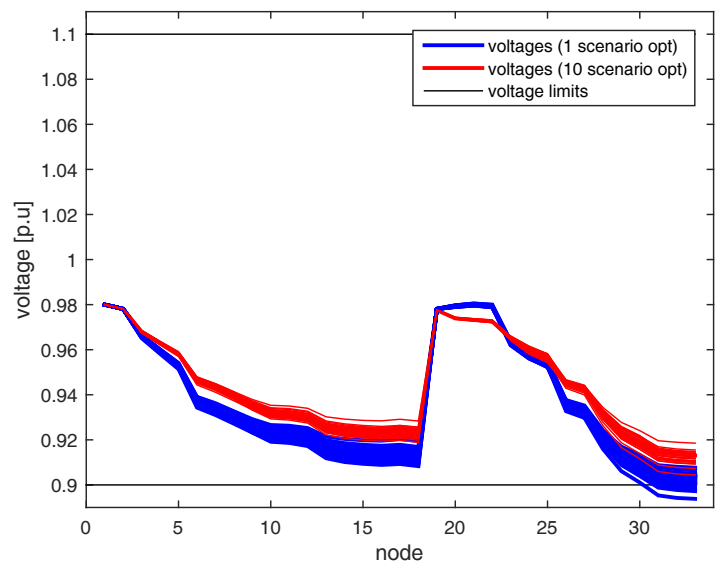

Figure 11: Voltages for 25 random scenarios 
Table 4: Optimal setpoints. $\left(\left.F F P_{n}^{*}\right|_{n=1},\left.F F Q_{n}^{*}\right|_{n=1}\right)=(2500 \mathrm{~kW}, 1500 \mathrm{kvar})$ Number of scenarios $=1$ Computation time $=1.6 \mathrm{~s}$

\begin{tabular}{|c|c|c|c|c|c|c|}
\hline Gen & Type & Status & $\begin{array}{l}P_{n}^{G^{*}} \\
{[\mathrm{~kW}]}\end{array}$ & $\begin{array}{l}Q_{n}^{G^{*}} \\
{[\mathrm{kvar}]}\end{array}$ & $\begin{array}{l}F F P_{n}^{*} \\
{[\mathrm{~kW}]}\end{array}$ & $\begin{array}{l}F F Q_{n}^{*} \\
{[\mathrm{kvar}]}\end{array}$ \\
\hline 2 & $\mathrm{FFC}$ & $\mathrm{ON}$ & - & - & 2500 & 1500 \\
\hline 6 & $\mathrm{FFC}$ & $\mathrm{OFF}$ & - & - & - & - \\
\hline 8 & PV & $\mathrm{ON}$ & - & 40 & - & - \\
\hline 11 & UPC & ON & 200 & 100 & - & - \\
\hline 12 & $\mathrm{PV}$ & ON & - & 15 & - & - \\
\hline 14 & $\mathrm{PV}$ & $\mathrm{ON}$ & - & 45 & - & - \\
\hline 17 & UPC & $\mathrm{ON}$ & 250 & 125 & - & - \\
\hline 21 & UPC & $\mathrm{ON}$ & 400 & 116.2 & - & - \\
\hline 24 & $\mathrm{PV}$ & $\mathrm{ON}$ & - & 100 & - & - \\
\hline 25 & UPC & $\mathrm{OFF}$ & - & - & - & - \\
\hline 26 & $\mathrm{FFC}$ & $\mathrm{ON}$ & - & - & 669.2 & 801.2 \\
\hline 29 & UPC & $\mathrm{ON}$ & 300 & 150 & - & - \\
\hline 30 & $\mathrm{PV}$ & $\mathrm{ON}$ & - & 75 & - & - \\
\hline 32 & PV & $\mathrm{ON}$ & - & 75 & - & - \\
\hline \multicolumn{7}{|c|}{ Number of scenarios $=10$} \\
\hline Gen & Type & Status & $\begin{array}{l}P_{n}^{G^{*}} \\
{[\mathrm{~kW}]}\end{array}$ & $\begin{array}{l}Q_{n}^{G^{*}} \\
{[\mathrm{kvar}]}\end{array}$ & $\begin{array}{l}F F P_{n}^{*} \\
{[\mathrm{~kW}]}\end{array}$ & $\begin{array}{l}F F Q_{n}^{*} \\
{[\mathrm{kvar}]}\end{array}$ \\
\hline 2 & $\mathrm{FFC}$ & $\mathrm{ON}$ & - & - & 2500 & 1500 \\
\hline 6 & FFC & $\mathrm{ON}$ & - & - & 1384.1 & 745.4 \\
\hline 8 & PV & $\mathrm{ON}$ & - & 40 & - & - \\
\hline 11 & UPC & $\mathrm{ON}$ & 200 & 100 & - & - \\
\hline 12 & $\mathrm{PV}$ & $\mathrm{ON}$ & - & 15 & - & - \\
\hline 14 & PV & $\mathrm{ON}$ & - & 45 & - & - \\
\hline 17 & UPC & $\mathrm{ON}$ & 250 & 125 & - & - \\
\hline 21 & UPC & OFF & - & - & - & - \\
\hline 24 & PV & $\mathrm{ON}$ & - & 100 & - & - \\
\hline 25 & UPC & OFF & - & - & - & - \\
\hline 26 & FFC & $\mathrm{ON}$ & - & - & 618.7 & 662.9 \\
\hline 29 & UPC & $\mathrm{ON}$ & 300 & 150 & - & - \\
\hline 30 & $\mathrm{PV}$ & $\mathrm{ON}$ & - & 75 & - & - \\
\hline 32 & PV & $\mathrm{ON}$ & - & 75 & - & - \\
\hline
\end{tabular}




\subsection{Scalability}

In order to test the possible scalability of the proposed optimization problem, a larger test case have been tested. Particularly, the case used is the 475141 node test feeder presented in [28], which is a $12.5 \mathrm{kV}$ radial distribution feeder located in Caracas. In addition, we have included 3 FFC generators (nodes 2, 7 and 15), 15 UPC generators (distributed along the whole network) and $12 \mathrm{PV}$ generators also distributed. Figure 12 shows the probability density function of optimization time (10 scenarios are considered). It has been obtained after launching 150 optimizations. Compared with the time required for the 33 node test feeder (Figure 9 in green), it can be observed the computational time is increased by 3 times. From this result, one could conclude the scalability of the proposed optimization problem and its resolution algorithm could be limited. Nevertheless, the time required can be reduced in two ways: i) by reducing the number of scenarios and ii) by changing the algorithm termination criteria. These actions could lead to a small degradation of the optimization objective and the system performance. Nevertheless, the optimization time in the case study is still reasonable.

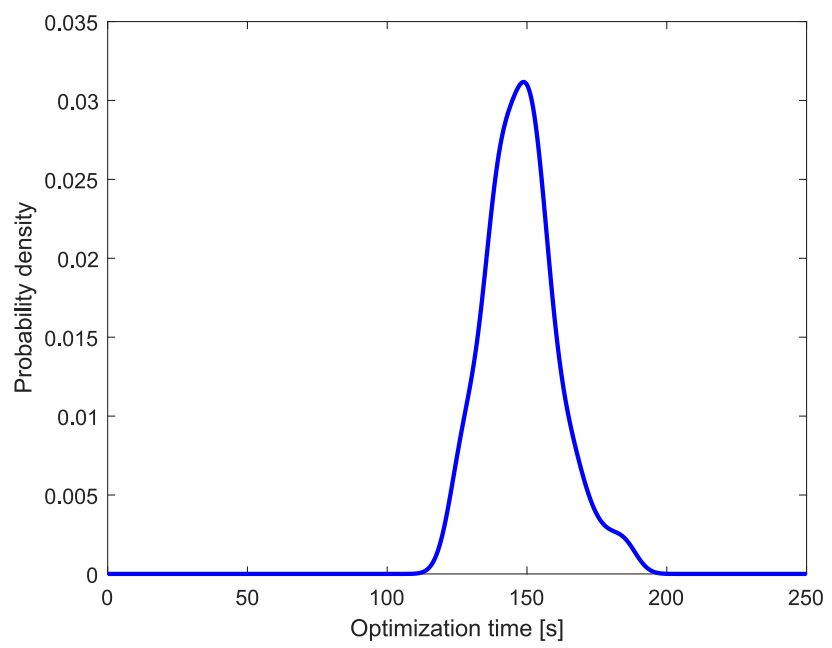

Figure 12: Probability density function of the optimization time for different number of scenarios (Kernel distribution). Case 141 nodes

\section{Conclusions}

This paper has presented a novel optimal power flow problem formulation for feeder flow controlled microgrids based on the second order cone 
relaxation of the DistFlow equations, which has been named OFFP-w.

Additionally, and in contrast to other studies which try to determine the objective function requirements to ensure the solution of the relaxed problem is also the solution of the non-relaxed problem, this paper presents a novel algorithm for solving the relaxed problem to ensure finding a meaningful solution without imposing conditions into the objective function.

In the particular case of feeder flow controlled microgrids, uncertainty may not only lead to a voltage or current limits violation, but also can produce the loss of the optimal operation point. The presented stochastic formulation permits finding a better solution improving the average objective function while maintaining the system voltages and currents within their limits. The better performance of the objective function mainly comes from the feeder flow setpoints penalties. The presented improvement can be even greater if the cost of violating the feeder flow constraints increases.

One of the limitations of the proposed optimization could be the scalability. Nevertheless, it has been tested in using a real 141 node test feeder with a reasonable computational time.

\section{Acknowledgements}

This work has been supported by the European Union's Horizon 2020 programme RESOLVD under the grant agreement number 773715. M. AragüésPeñalba and E. Prieto-Araujo are lecturers of the Serra Húnter Programme.

\section{Appendix A: Data used in the case study}

The IEEE 33-Bus distribution system data used in this paper is shown in Tables .5 and .6 . The rest of the parameters of the base case are shown in Table 7. The costs of importing energy from the grid (the cost of generator 1, i.e. the slack node) is obtained based on the Spanish market costs at 12.6 $\mathrm{kV}$. It has been considered the energy cost, the grid access tariffs and taxes.

\section{References}

[1] J. M. Ivan Pineda, Sarah Azau, J. Wilkes, Wind in power. 2013 European statistics, EWEA, European Wind Energy Association, 2014. 
Table .5: Bus data

\begin{tabular}{|c|c|c|c|c|c|c|c|c|c|}
\hline Bus Id & $\begin{array}{l}P^{C} \\
{[k W]}\end{array}$ & $\begin{array}{l}Q^{C} \\
{[\text { kvar }]}\end{array}$ & $\begin{array}{l}P^{G \max } \\
{[\mathrm{kW}]}\end{array}$ & $\begin{array}{l}P^{G m i n} \\
{[k W]}\end{array}$ & $\begin{array}{l}Q^{\text {Gmax }} \\
{[\text { kvar }]}\end{array}$ & $\begin{array}{l}Q^{\text {Gmin }} \\
{[k v a r]}\end{array}$ & $\begin{array}{l}C^{a g} \\
{[€ / h]}\end{array}$ & $\begin{array}{l}C^{b g} \\
{[€ / k W h]}\end{array}$ & $\begin{array}{l}C^{c g} \\
{[€ / k W h]}\end{array}$ \\
\hline 1 & 0 & 0 & 999999 & -999999 & 999999 & -999999 & 0.000 & 0.103 & 0 \\
\hline 2 & 100 & 60 & 1500 & 450 & 750 & -750 & 14.530 & 0.083 & 0.0001 \\
\hline 3 & 90 & 40 & 0 & 0 & 0 & 0 & 0 & 0 & 0 \\
\hline 4 & 120 & 80 & 0 & 0 & 0 & 0 & 0 & 0 & 0 \\
\hline 5 & 60 & 30 & 0 & 0 & 0 & 0 & 0 & 0 & 0 \\
\hline 6 & 60 & 20 & 800 & 270 & 400 & -400 & 14.530 & 0.103 & 0.0001 \\
\hline 7 & 200 & 100 & 0 & 0 & 0 & 0 & 0 & 0 & 0 \\
\hline 8 & 200 & 100 & 80 & 0 & 0 & 0 & 0 & 0 & 0 \\
\hline 9 & 60 & 20 & 0 & 0 & 0 & 0 & 0 & 0 & 0 \\
\hline 10 & 60 & 20 & 0 & 0 & 0 & 0 & 0 & 0 & 0 \\
\hline 11 & 45 & 30 & 200 & 70 & 100 & -100 & 5.080 & 0.035 & 0.0002 \\
\hline 12 & 60 & 35 & 30 & 0 & 0 & 0 & 0 & 0 & 0 \\
\hline 13 & 60 & 35 & 0 & 0 & 0 & 0 & 0 & 0 & 0 \\
\hline 14 & 120 & 80 & 90 & 0 & 0 & 0 & 0 & 0 & 0 \\
\hline 15 & 60 & 10 & 0 & 0 & 0 & 0 & 0 & 0 & 0 \\
\hline 16 & 60 & 20 & 0 & 0 & 0 & 0 & 0 & 0 & 0 \\
\hline 17 & 60 & 20 & 250 & 80 & 125 & -125 & 5.080 & 0.035 & 0.0002 \\
\hline 18 & 90 & 40 & 0 & 0 & 0 & 0 & 0 & 0 & 0 \\
\hline 19 & 90 & 40 & 0 & 0 & 0 & 0 & 0 & 0 & 0 \\
\hline 20 & 90 & 40 & 0 & 0 & 0 & 0 & 0 & 0 & 0 \\
\hline 21 & 90 & 40 & 400 & 130 & 200 & -200 & 0.851 & 0.069 & 0.0002 \\
\hline 22 & 90 & 40 & 0 & 0 & 0 & 0 & 0 & 0 & 0 \\
\hline 23 & 90 & 50 & 0 & 0 & 0 & 0 & 0 & 0 & 0 \\
\hline 24 & 420 & 200 & 200 & 0 & 0 & 0 & 0 & 0 & 0 \\
\hline 25 & 420 & 200 & 200 & 70 & 100 & -100 & 0.851 & 0.069 & 0.0002 \\
\hline 26 & 60 & 25 & 700 & 230 & 350 & -350 & 14.530 & 0.103 & 0.0001 \\
\hline 27 & 60 & 25 & 0 & 0 & 0 & 0 & 0 & 0 & 0 \\
\hline 28 & 60 & 20 & 0 & 0 & 0 & 0 & 0 & 0 & 0 \\
\hline 29 & 120 & 70 & 300 & 100 & 150 & -150 & 3.405 & 0.013 & 0.0002 \\
\hline 30 & 200 & 600 & 150 & 0 & 75 & -75 & 0 & 0 & 0 \\
\hline 31 & 150 & 70 & 0 & 0 & 0 & 0 & 0 & 0 & 0 \\
\hline 32 & 210 & 100 & 150 & 0 & 0 & 0 & 0 & 0 & 0 \\
\hline 33 & 60 & 40 & 0 & 0 & 0 & 0 & 0 & 0 & 0 \\
\hline
\end{tabular}

[2] F. Rafi, M. Hossain, J. Lu, Hierarchical controls selection based on pv penetrations for voltage rise mitigation in a lv distribution network, International Journal of Electrical Power \& Energy Systems 81 (2016) 123 - 139. doi:https://doi.org/10.1016/j.ijepes.2016.02.013.

[3] C. S. aegerl, L. Tao, The Microgrids Concept, John Wiley and sons, 2013.

[4] A. Ulbig, Borsche, T. S., G. Andersson, Impact of low rotational inertia 530 on power system stability and operation, in: IFAC World Congress 2014, Capetown, South Africa.

[5] A. Gholizadeh, A. Rabiee, R. Fadaeinedjad, A scenario-based 
Table .6: Line data

\begin{tabular}{lllll|lllll}
\hline From bus & To bus & $\mathrm{r}[\Omega]$ & $\mathrm{x}[\Omega]$ & $i^{\max }\left[A^{2}\right]$ & From bus & To bus & $\mathrm{r}[\Omega]$ & $\mathrm{x}[\Omega]$ & $i^{\max }\left[A^{2}\right]$ \\
\hline 1 & 2 & 0.0922 & 0.0470 & $10^{6}$ & 17 & 18 & 0.7320 & 0.5740 & $10^{6}$ \\
2 & 3 & 0.4930 & 0.2511 & $10^{6}$ & 2 & 19 & 0.1640 & 0.1565 & $10^{6}$ \\
3 & 4 & 0.3660 & 0.1864 & $10^{6}$ & 19 & 20 & 1.5042 & 1.3554 & $10^{6}$ \\
4 & 5 & 0.3811 & 0.1941 & $10^{6}$ & 20 & 21 & 0.4095 & 0.4784 & $10^{6}$ \\
5 & 6 & 0.8190 & 0.7070 & $10^{6}$ & 21 & 22 & 0.7089 & 0.9373 & $10^{6}$ \\
6 & 7 & 0.1872 & 0.6188 & $10^{6}$ & 3 & 23 & 0.4512 & 0.3083 & $10^{6}$ \\
7 & 8 & 0.7114 & 0.2351 & $10^{6}$ & 23 & 24 & 0.8980 & 0.7091 & $10^{6}$ \\
8 & 9 & 1.0300 & 0.7400 & $10^{6}$ & 24 & 26 & 0.8960 & 0.7011 & $10^{6}$ \\
9 & 10 & 1.0440 & 0.7400 & $10^{6}$ & 6 & 26 & 0.2030 & 0.1034 & $10^{6}$ \\
10 & 11 & 0.1966 & 0.0650 & $10^{6}$ & 26 & 27 & 0.2842 & 0.1447 & $10^{6}$ \\
11 & 12 & 0.3744 & 0.1238 & $10^{6}$ & 27 & 28 & 1.0590 & 0.9337 & $10^{6}$ \\
12 & 13 & 1.4680 & 1.1550 & $10^{6}$ & 28 & 29 & 0.8042 & 0.7006 & $10^{6}$ \\
13 & 14 & 0.5416 & 0.7129 & $10^{6}$ & 29 & 30 & 0.5075 & 0.2585 & $10^{6}$ \\
14 & 15 & 0.5910 & 0.5260 & $10^{6}$ & 30 & 31 & 0.9744 & 0.9630 & $10^{6}$ \\
15 & 16 & 0.7463 & 0.5450 & $10^{6}$ & 31 & 32 & 0.3105 & 0.3619 & $10^{6}$ \\
16 & 17 & 1.2890 & 1.7210 & $10^{6}$ & 32 & 33 & 0.3410 & 0.5302 & $10^{6}$ \\
\hline
\end{tabular}

voltage stability constrained planning model for integration of large-scale wind farms, International Journal of Electrical

535 Power \& Energy Systems 105 (2019) 564 - 580. doi:https: //doi.org/10.1016/j.ijepes.2018.09.002.

URL http://wWW.sciencedirect.com/science/article/pii/ S0142061518300255

[6] A. Cabrera-Tobar, E. Bullich-Massagué, M. Aragüés-Peñalba,

O. Gomis-Bellmunt, Review of advanced grid requirements for the integration of large scale photovoltaic power plants in the transmission system, Renewable and Sustainable Energy Rev. 62 (2016) 971 987. doi:https://doi.org/10.1016/j.rser.2016.05.044.

[7] E. Bullich-Massagué, R. Ferrer-San-José, M. Aragüés-Peñalba, 545 L. Serrano-Salamanca, C. Pacheco-Navas, O. Gomis-Bellmunt, Power plant control in large-scale photovoltaic plants: design, implementation and validation in a $9.4 \mathrm{mw}$ photovoltaic plant, IET Renewable Power Generation 10 (1) (2016) 50-62. doi:10.1049/iet-rpg.2015.0113.

[8] K.-L. Nguyen, D.-J. Won, S.-J. Ahn, I.-Y. Chung, Power sharing method for a grid connected microgrid with multiple distributed generators, Journal of Electrical Engineering and Technology 7 (4) (2012) 459 467. doi:10.5370/JEET.2012.7.4.459.

[9] P. Piagi, R. H. Lasseter, Autonomous control of microgrids, in: 2006 
Table .7: Additional parameters

\begin{tabular}{ll}
\hline Parameter & Value \\
\hline$v^{\text {max }}[p . u]$. & $1.21^{*}$ \\
$v^{\text {min }}[p . u]$. & $0.81^{*}$ \\
$v^{*}[p . u]$. & 1 \\
$C^{a f f p}, C^{\text {affq }}[€]$ & 0 \\
$C^{b f f p}[€ / k W]$ & 2.4 \\
$C^{b f f q}[€ / k V a r]$ & 2.4 \\
$M_{1}, M_{2}, M_{3}$ & $10^{* *}$ \\
$\Delta C^{\prime}[€]$ & Depends on the \\
$N_{s}($ number of scenarios $)$ & 10 \\
$\left.F F P_{n}^{*}\right|_{n=1}[k W]$ & 750 \\
$\left.F F Q_{n}^{*}\right|_{n=1}[k V a r]$ & 500 \\
\hline$v^{\text {max }}$ and $v^{\text {min }}$ is square voltage. It is equivalent to \\
$0.9 \leq V \leq 1.1$ \\
$* *$ Is large enough considering that the formulation has been \\
done in p.u. $S_{\text {Base }}=1[M V A], V_{\text {base }}=12.66[k V]$
\end{tabular}

IEEE Power Engineering Society General Meeting, 2006, pp. 8 pp.-doi:10.1109/PES.2006.1708993.

[10] S. J. Ahn, J. W. Park, I. Y. Chung, S. I. Moon, S. H. Kang, S. R. Nam, Power-sharing method of multiple distributed generators considering control modes and configurations of a microgrid, IEEE Trans. on Power Delivery 25 (3) (2010) 2007-2016. doi:10.1109/TPWRD.2010.2047736.

560

口

[11] V. R. Pandi, A. Al-Hinai, A. Feliachi, Adaptive coordinated feeder flow control in distribution system with the support of distributed energy resources, International Journal of Electrical Power \& Energy Systems 85 (2017) 190 - 199. doi:http://dx.doi.org/10.1016/j.ijepes. 2016. 09.004 .

[12] V. R. Pandi, A. Al-Hinai, A. Feliachi, Coordinated control of distributed energy resources to support load frequency control, Energy Conversion and Management 105 (2015) 918 - 928. doi:http://dx.doi.org/10. $1016 /$ j.enconman.2015.08.046. 
[13] S. J. Ahn, S. R. Nam, J. H. Choi, S. I. Moon, Power scheduling of distributed generators for economic and stable operation of a microgrid, IEEE Trans. on Smart Grid 4 (1) (2013) 398-405. doi:10.1109/TSG. 2012.2233773.

[14] Z. xia Xiao, J. M. Guerrero, J. Shuang, D. Sera, E. Schaltz, J. C. Vsquez, Flat tie-line power scheduling control of grid-connected hybrid microgrids, Applied Energydoi:https://doi.org/10.1016/j. apenergy.2017.07.066.

[15] R. Majumder, A. Ghosh, G. Ledwich, F. Zare, Power management and power flow control with back-to-back converters in a utility connected microgrid, IEEE Trans. on Power Systems 25 (2) (2010) 821-834. doi: 10.1109/TPWRS. 2009.2034666.

[16] M. Farivar, S. H. Low, Branch flow model: Relaxations and convexification - part i, IEEE Trans. on Power Systems 28 (3) (2013) 2554-2564. doi:10.1109/TPWRS.2013.2255317.

[17] B. P. Swaminathan, Operational Planning of Active Distribution Networks - Convex Relaxation under Uncertainty, Theses, Université Grenoble Alpes (Sep. 2017).

[18] Y. Levron, J. M. Guerrero, Y. Beck, Optimal power flow in microgrids with energy storage, IEEE Transactions on Power Systems 28 (3) (2013) 3226-3234. doi:10.1109/TPWRS.2013.2245925.

590 [19] R. Palma-Behnke, C. Benavides, F. Lanas, B. Severino, L. Reyes, J. Llanos, D. Sez, A microgrid energy management system based on the rolling horizon strategy, IEEE Transactions on Smart Grid 4 (2) (2013) 996-1006. doi:10.1109/TSG. 2012.2231440.

[20] N. Holjevac, T. Capuder, N. Zhang, I. Kuzle, C. Kang, Cor$595 \square \quad$\begin{tabular}{llllll} 
rective & receding horizon scheduling of flexible distributed multi- \\
\hline energy & microgrids, Applied Energy & 207 & (2017) & 176 & -
\end{tabular} transformative Innovations for a Sustainable Future Part II. doi:https://doi.org/10.1016/j.apenergy.2017.06.045. URL http://ww. sciencedirect.com/science/article/pii/ S0306261917307973 
[21] M. Baran, F. F. Wu, Optimal sizing of capacitors placed on a radial distribution system, IEEE Trans. on Power Delivery 4 (1) (1989) 735743. doi:10.1109/61.19266.

[22] S. Touré, Networks optimization : active and flexible network, Theses,

605 Université de Grenoble (Oct. 2014). URL https://tel.archives-ouvertes.fr/tel-01122254

[23] M. E. Baran, F. F. Wu, Network reconfiguration in distribution systems for loss reduction and load balancing, IEEE Trans. on Power Delivery 4 (2) (1989) 1401-1407. doi:10.1109/61.25627.

[24] A. Maulik, D. Das, Optimal operation of droop-controlled islanded microgrids, IEEE Transactions on Sustainable Energy 9 (3) (2018) 13371348. doi:10.1109/TSTE.2017.2783356.

[25] C. Monteiro, T. Santos, L. A. Fernandez-Jimenez, I. J. Ramirez-Rosado, M. S. Terreros-Olarte, Short-term power forecasting model for photovoltaic plants based on historical similarity, Energies 6 (5) (2013) 26242643. doi:10.3390/en6052624.

[26] D. P. Larson, L. Nonnenmacher, C. F. Coimbra, Day-ahead forecasting of solar power output from photovoltaic plants in the american southwest, Renewable Energy 91 (2016) 11 - 20. doi:https://doi.org/10.

1016/j.renene.2016.01.039.

[27] H. Chitsaz, H. Shaker, H. Zareipour, D. Wood, N. Amjady, Short-term electricity load forecasting of buildings in microgrids, Energy and Buildings 99 (2015) 50 - 60. doi:https://doi.org/10.1016/j.enbuild. 2015.04.011.

[28] H. Khodr, F. Olsina, P. D. O.-D. Jesus, J. Yusta, Maximum savings approach for location and sizing of capacitors in distribution systems, Electric Power Systems Research 78 (7) (2008) 1192 - 1203. doi:https://doi.org/10.1016/j.epsr.2007.10.002. URL http://www.sciencedirect.com/science/article/pii/ S0378779607002143 